\title{
Nuclear targeting of the serine protease granzyme A (fragmentin-1)
}

\author{
David A. Jans ${ }^{1, *}$, Lyndall J. Briggs ${ }^{1}$, Patricia Jans ${ }^{1}$, Christopher J. Froelich ${ }^{4}$, Gayathri Parasivam ${ }^{3}$, \\ Sharad Kumar' ${ }^{3}$, Vivien R. Sutton ${ }^{2}$ and Joseph A. Trapani ${ }^{2}$ \\ ${ }^{1}$ Nuclear Signalling Laboratory, Division for Biochemistry and Molecular Biology, John Curtin School of Medical Research, PO Box \\ 334, Canberra City, ACT 2601, Australia \\ ${ }^{2}$ Cellular Cytotoxicity Laboratory, The Austin Research Institute, Heidelberg, Australia \\ ${ }^{3}$ Hanson Centre for Cancer Research, Adelaide, Australia \\ ${ }^{4}$ Department of Medicine, Northwestern University Medical School, Evanston, USA \\ *Author for correspondence (e-mail: david.jans@anu.edu.au)
}

Accepted 29 June; published on WWW 13 August 1998

\section{SUMMARY}

Cytolytic granule-mediated target cell killing is effected in part through synergistic action of the membrane-acting protein perforin and serine proteases such as granzymes $\mathrm{A}$ (GrA) or B (GrB). In the present study we examine GrA cellular entry and nuclear uptake in intact mouse myeloid FDC-P1 cells exposed to perforin using confocal laser scanning microscopy, as well as reconstitute $\operatorname{GrA}$ nuclear uptake in vitro. GrA alone was found to be able to enter the cytoplasm of intact cells but did not accumulate in nuclei. In the presence of perforin, it specifically accumulated in the cell nuclei, with maximal levels about 2.5 times those in the cytoplasm after 2.5 hours. In vitro, GrA accumulated in the nucleus and nucleolus maximally to levels that were fourand sixfold, respectively, those in the cytoplasm. In contrast, the active form of the apoptotic cysteine protease CPP32 did not accumulate in nuclei in vitro. Nuclear/nucleolar import of GrA in vitro was independent of ATP and not inhibitable by the non-hydrolyzable GTP analog GTP $\gamma \mathrm{S}$, but was dependent on exogenously added cytosol. Importantly, GrA was found to be able to accumulate in the nucleus of semiintact cells in the presence of the nuclear envelopepermeabilizing detergent CHAPS, implying that the mechanism of nuclear accumulation was through binding to insoluble factors in the nucleus. GrB was found for the first time to be similar in this regard. The results support the contention that GrA and GrB accumulate in the nucleus through a novel nuclear import pathway, and that this is integral to induction of the nuclear changes associated with cytolytic granule-mediated apoptosis.

Key words: Apoptosis, Granzyme, Perforin, Nuclear localization, Confocal laser scanning microscopy, Mouse

\section{INTRODUCTION}

One major active component released by cytotoxic natural killer (NK) and T cells (CTLs) during degranulation is perforin (Henkart et al., 1984), which can damage cells through membrane perturbation. Target cell DNA fragmentation and chromatin condensation, which are characteristic of apoptosis (Earnshaw, 1995), require additional granule components, serine proteases termed granzymes, which synergise with perforin to effect apoptosis. Of the eight granzymes identified in the mouse, the most abundant is granzyme A (GrA or fragmentin-1), a $65 \mathrm{kDa}$ homodimer which, in contrast to the monomeric $32 \mathrm{kDa}$ granzyme $\mathrm{B}$ (GrB or fragmentin-2), is able to activate IL-1 $\beta$ directly (Irmler et al., 1995), as well as to cleave various intracellular/extracellular proteins, including the thrombin receptor (Suidan et al., 1994) and the nuclear/nucleolar protein nucleolin (Pasternack et al., 1991). GrA and GrB differ in their substrate specificities; whereas GrA cleaves at basic amino acid residues, GrB is an aspase, cleaving at aspartic acid residues (Poe et al., 1991).

In combination with perforin, either purified $\mathrm{GrA}$ or $\mathrm{GrB}$ is able to effect DNA fragmentation, although GrA/perforinmediated cell killing exhibits slower kinetics (Hayes et al., 1989; Shi et al., 1992a,b). CTLs from GrB knock-out mice have a severely impaired capacity to cause apoptosis in shortterm assays (Heusel et al., 1994), but those from GrA knockout mice do not, implying that GrA does not have an indispensable role in the induction of apoptosis in target cells by NK cells and alloreactive CTL (Ebnet et al., 1995). GrBdeficient CTLs display nearly normal levels of cytolytic activity after prolonged incubation with target cells (Heusel et al., 1994), however, indicating that GrA may be involved in inducing target cell apoptosis via a mechanism triggered after prolonged incubation of killer and target cells, in cells that are relatively resistant to $\mathrm{GrB}$, or in the absence of $\mathrm{GrB}$ (see also Shi et al., 1992a,b). Further differences between GrA and GrB include the fact that GrA nucleolytic activity can be partially suppressed by cycloheximide, which is not the case for GrB (Ebnet et al., 1995), and evidence that GrA has a direct role in triggering chromosome degradation during apoptosis (Munger et al., 1989; Hayes et al., 1989).

We have recently demonstrated that nuclear accumulation of 
$\mathrm{GrB}$ in intact cells in the presence of perforin occurs prior to the nuclear events of apoptosis (Trapani et al., 1998), implying that nuclear translocation of granzymes may constitute the means by which the apoptotic signal is communicated to the nucleus. We wished to assess whether GrA, in view of its clearly quite distinct role from that of $\mathrm{GrB}$ with respect to apoptotic functions, as well as its very different structure and substrate specificity, may also translocate to the nucleus prior to apoptosis. Therefore we set out to examine the entry and distribution of GrA in intact cells exposed to perforin using quantitative confocal laser scanning microscopy (CLSM). Our results demonstrate that GrA can enter the cytoplasm independently of perforin, but that the co-application of perforin effects a rapid redistribution of GrA to the nucleus, which is followed by the induction of apoptosis. Nuclear uptake of GrA could be reconstituted in vitro, and was shown to be dependent on cytosolic factors, but independent of ATP and not inhibited by non-hydrolyzable GTP analogs. The mechanism of nuclear accumulation appeared to be through binding to insoluble factors in the nucleus, implying the existence of a novel nuclear import pathway. The findings are consistent with the idea that nuclear uptake of granzymes may be central to eliciting the nuclear changes of apoptosis in target cells.

\section{MATERIALS AND METHODS}

\section{Chemicals and reagents}

Specific antibodies to nucleolin (C23), active in western blotting with HTC cell extracts (Jans et al., 1996), were provided by Harris Busch (Baylor College, Houston, Texas). Isopropyl- $\beta$-thiogalactoside (IPTG), 5(6)-carboxyfluorescein-N-hydroxysuccinimide ester (FLUOS) and the detergent 3[(3-cholamidopropyl)-dimethylamino]1-propane-sulfonate (CHAPS) were from Boehringer-Mannheim; fluorescein isothiocyanate (FITC) and 5-iodacetamido-fluorescein (IAF) were from Molecular Probes. Other reagents were from the sources previously described (Jans et al., 1991, 1996; Trapani et al., 1996, 1998).

\section{Cell culture}

Cells of the HTC rat hepatoma tissue culture cell line, a derivative of Morris hepatoma 7288C, were cultured in Dulbecco's Modified Eagle's Medium (DMEM) supplemented with $10 \%$ foetal calf serum (FCS) as described previously (Rihs et al., 1991; Jans et al., 1991). Mouse FDC-P1 myeloid cells were cultured in DMEM supplemented with $10 \%$ FCS and recombinant IL-3-containing culture supernatant (Karasuyama and Melchers, 1988).

\section{Protein purification and labelling}

Purification of GrA was performed as described previously (Hanna et al., 1996). Immunoaffinity purification of human GrB from nuclear extracts of YT cells was performed as described (Trapani et al., 1993, 1996). Human perforin was isolated using affinity chromatography as described previously (Froehlich et al., 1996b,c). Recombinant Histagged CPP32 (Caspase-3) protein (active form) was expressed in Escherichia coli strain BL21(DE3) from the pET21G expression construct, kindly provided by Dr E. Alnemri. 1 mM IPTG was used to induce protein expression, prior to purification by affinity chromatography using Talon resin (Clontech) and elution with 200 mM EDTA. CPP32 enzymic activity was assayed using Asp-Glu-ValAsp-7-amino-4-trifluoromethyl coumarin as a substrate (Enzyme Systems Inc., Dublin, Ca) as previously described (Harvey et al., 1997). Recombinant cyclin dependent kinase (cdk) subunit $\mathrm{p} 13^{\text {sucl }}$ was expressed in E. coli, purified and labelled with FLUOS as described (Trapani et al., 1998). GrA, GrB and CPP32 were labelled with FITC in the absence of reducing agents as described previously for GrB (Trapani et al., 1996). FITC-GrA was a dimer (65 kDa), as indicated by western blotting after non-denaturing polyacrylamide gel electrophoresis.

The SV40 large tumor-antigen (T-ag) fusion protein T-ag-CcN- $\beta$ Gal (Rihs et al., 1991), used as a control for nuclear localization signal (NLS)-dependent nuclear import, contains T-ag amino acids 111-135, including the NLS, fused N-terminal to the E. coli $\beta$-galactosidase coding sequence (amino acids 9-1023). Protein expression was induced by IPTG in E. coli and labelling with IAF performed as described previously (Rihs et al., 1991).

\section{Cellular uptake and distribution of granzymes}

FDC-P1 whole cell uptake and subcellular transport of labelled GrA were examined as described for GrB (Trapani et al., 1998). $6 \mu$ of logarithmic phase cells $\left(4 \times 10^{6}\right.$ cells $\left./ \mathrm{ml}\right)$ in Hank's buffered saline solution containing $10 \mathrm{mM}$ Hepes pH 7.2, $2 \mathrm{mM} \mathrm{CaCl}_{2}, 0.4 \%$ BSA and $0.1 \%(\mathrm{v} / \mathrm{v})$ IL-3-containing culture supernatant were incubated for the specified times with an equal volume of perforin (100-1000 units/ml, final concentration) and/or FITC-GrA (2.5-10 $\mu \mathrm{g} / \mathrm{ml}$, final concentration) diluted and mixed in $10 \mathrm{mM}$ Hepes, $150 \mathrm{mM} \mathrm{NaCl}, 1$ mM EGTA, pH 7.2, immediately before the assay. The reaction mixture was immediately pipetted onto a glass slide, which was then sealed, prior to incubation at $37^{\circ} \mathrm{C}$ and imaging of fluorescence by CLSM at various times using a Bio-Rad MRC-600 CLSM (Jans et al., 1996). The dose of perforin used elicited $<5 \%$ specific ${ }^{51} \mathrm{Cr}$ release from FDC-P1 cells ( 4 hour assay at $37^{\circ} \mathrm{C}$ ); FITC-dextran had no effect on ${ }^{51} \mathrm{Cr}$ release from FDC-P1 cells incubated with or without perforin (Trapani et al., 1998). That the dimeric form of GrA was $>85 \%$ maintained in its active form even after $>60$ minutes incubation with either FDC lysate or whole cells in the presence of perforin was demonstrated by western blotting and measurement of BLT esterase activity (not shown).

\section{Apoptosis}

Apoptotic morphology was scored using visual criteria as previously (Trapani et al., 1998). The validity of this approach has been confirmed using other techniques such as TUNEL analysis and annexin V expression (Trapani et al., 1998), as well as by propidium iodide staining and electron microscopy (not shown).

\section{In vitro nuclear transport assay}

Analysis of nuclear import kinetics at the single cell level was performed using mechanically perforated HTC cells in conjunction with CLSM (Jans et al., 1991, 1996). Assays were routinely carried out in intracellular buffer $(110 \mathrm{mM} \mathrm{KCl}, 5 \mathrm{mM} \mathrm{NaHCO} 3,5 \mathrm{mM}$ $\mathrm{MgCl}_{2}, 1 \mathrm{mM}$ EGTA, $0.1 \mathrm{mM} \mathrm{CaCl}$, $20 \mathrm{mM}$ Hepes, $1 \mathrm{mM}$ DTT, $\mathrm{pH}$ 7.4) in the presence of cytosolic extract (untreated reticulocyte lysate), an ATP regenerating system $(0.125 \mathrm{mg} / \mathrm{ml}$ creatine kinase, $30 \mathrm{mM}$ creatine-phosphate, $2 \mathrm{mM}$ ATP), and transport substrate $(30-40 \mu \mathrm{g} / \mathrm{ml}$ FITC-labelled granzymes or dextran), and nuclear uptake followed with CLSM (Jans et al., 1996; Trapani et al., 1996). Where the ATP dependence of transport was tested, apyrase pretreatment was used to hydrolyze ATP present in cytosolic extracts $(10$ minutes at room temperature with 800 units $/ \mathrm{ml})$ and perforated cells $(15$ minutes at $37^{\circ} \mathrm{C}$ with 0.2 units $/ \mathrm{ml}$ ) (Newmeyer and Forbes, 1988), and assays performed in the absence of the ATP regenerating system (Jans et al., 1996). In experiments where the dependence of transport on the GTPbinding protein Ran/TC4 (Moore and Blobel, 1994) was tested, cytosolic extract was treated with $850 \mu \mathrm{M}$ GTP $\gamma \mathrm{S}$ (non-hydrolyzable GTP analog) for 5 minutes at room temperature, prior to use in the in vitro assay (Jans et al., 1996).

Nuclear and nucleolar accumulation of granzymes was also examined in vitro in the presence of a one tenth volume of $20 \mathrm{mM}$ Tris, $\mathrm{pH} 7.0$, containing $10 \%$ glycerol and $0.25 \%$ CHAPS, which 
results in permeabilization of the nuclear envelope; accumulation under these conditions only results from binding to nuclear components such as lamins, chromatin etc. (Efthymiadis et al., 1997, 1998).

\section{Image analysis}

Image analysis of CLSM images using the NIH Image 1.60 public domain software and curve fitting for data from the in vitro nuclear transport assay were carried out as described previously (Jans et al., 1996). Results for whole cell experiments were expressed in terms of cellular uptake $=\mathrm{Fc} /$ Fmedium (where $\mathrm{Fc}=$ fluorescence quantitated in the cytoplasm, relative to Fmedium, fluorescence quantitated in the medium, following subtraction of background fluorescence), and nuclear accumulation $=\mathrm{Fn} / \mathrm{c}$ (where $\mathrm{Fn}=$ fluorescence quantitated in the nucleus, relative to $\mathrm{Fc}$, following subtraction of background fluorescence) (Trapani et al., 1998).

\section{ELISA-based binding assay}

An ELISA-based binding assay (Efthymiadis et al., 1997, 1998; Hübner et al., 1997) was used to examine the binding affinity between importin subunits (mouse importin 58 and 97 glutathione-Stransferase (GST) fusion proteins, expressed as described; Efthymiadis et al., 1997; Hübner et al., 1997) and GrA. This involved coating 96-well microtiter plates with GrA, hybridization with increasing concentrations of importin subunits, and detection of bound importin-GST using goat anti-GST primary antibodies and alkaline phosphatase-coupled rabbit anti-goat secondary antibodies and the substrate $p$-nitrophenyl phosphate ( $p$-NPP) (Efthymiadis et al., 1997; Hübner et al., 1997). Absorbance measurements were performed over 90 minutes using a plate reader (Molecular Devices), and values corrected by subtracting absorbance values both at time $=$ 0 minutes and in wells incubated without importin (Efthymiadis et al., 1997; Hübner et al., 1997).

\section{RESULTS}

\section{Nuclear uptake of GrA in intact cells exposed to sublytic concentrations of perforin correlates with apoptosis}

GrA was labelled with FITC and cellular and nuclear uptake assessed in intact FDC-P1 cells in the absence or presence of sublytic concentrations of perforin at $37^{\circ} \mathrm{C}$ using CLSM (Fig. 1). Within 20-30 minutes, the cytoplasm and nucleus of about $70 \%$ of the cells became fluorescent, staining that was predictive of subsequent induction of apoptosis (Fig. 1B), whilst the remaining cells took up GrA in lower amounts and appeared to be spared from apoptosis. In the strongly staining population, GrA was localized principally within the nucleus as compared to the cytoplasm (Fig. 1A right panel, and see below). Neither population showed apoptotic features at early time points: the outline of the nucleus was smooth and regular, and fluorescence was distributed uniformly throughout the nuclei of the 'preapoptotic' cells. Cells exposed to FITC-GrA in the absence of perforin were similar in appearance to the weakly stained population exposed to both reagents. Only relatively small amounts of FITC-GrA were taken up into the cytoplasm by 10 minutes and corresponding extracellular fluorescence remained higher than in cells additionally exposed to perforin (Fig. 1, and see below). From about 20 minutes onwards, the numbers of apoptotic cells (defined by nuclear collapse or disintegration; Trapani et al., 1998) increased, reaching a plateau at about 50 minutes (Fig. 1B left panel).
Quantitation of fluorescence due to GrA uptake clearly indicated that GrA alone was able to enter the cytoplasm of intact FDC-P1 cells but that it did not accumulate in nuclei (Fig. 1A middle panel, B right panel). In the presence of sublytic concentrations of perforin, GrA was taken up by the cells more rapidly (readily detectable within about 10-15 minutes), and to a greater extent (about 8 times greater than in its absence at 100 minutes), as indicated by measurements of cellular uptake (Fc/Fmedium) (Fig. 1B middle panel, C). More significantly, GrA accumulated strongly in the cell nuclei in the presence of perforin, peaking at levels about 2.5 times those in the cytoplasm, with maximal accumulation being attained within about 35 minutes ( $\mathrm{t}_{1 / 2}$ was approx. 7.5 minutes; Fig. 1B right panel). The $\mathrm{Fn} / \mathrm{c}$ did not exceed 0.9 in non-apoptotic cells (Fig. 1B, right panel). The results thus indicated that whilst GrA is clearly able to enter cells in its absence, perforin induces much higher cytoplasmic uptake and, importantly, elicits nuclear accumulation of GrA and apoptosis.

In contrast to GrA, control molecules of a $20 \mathrm{kDa}$ FITClabelled dextran and the cdk subunit $\mathrm{p} 13^{\text {suc } 1}$ did not accumulate appreciably in intact cells in the presence of perforin even up to 3 hours (Fig. 1, and not shown). This shows that sublytic concentrations of perforin neither result in the formation of large membrane pores which would enable the direct entry of macromolecules such as cytolytic granule components into the cell (see Trapani et al., 1998), nor trigger the cellular uptake of extracellular molecules non-specifically. Rather, GrA would appear to be taken up by a specific process, and that a signalling event effected by perforin, presumably at the cell membrane, is required to bring about nuclear accumulation once GrA is inside the target cell. Cells exposed to perforin and FITCdextran or to FITC-GrA alone (Fig. 1B) showed fewer than 9\% apoptotic cells throughout.

\section{Nuclear targeting of GrA in vitro}

To address the question of the mechanism of GrA accumulation in the nucleus, we reconstituted GrA nuclear transport in vitro using our system of mechanically perforated HTC cells (Jans et al., 1991, 1996), which in identical fashion to the more widely used digitonin-permeabilised cell system (Adam et al., 1990; Adam and Gerace, 1991), relies on the addition of an ATP-regenerating system and exogenous cytosol for active, NLS-dependent nuclear protein import (Jans et al., 1991, 1996; see Materials and methods). GrA showed rapid accumulation in both nuclei and nucleoli (Fig. 2; Table 1); since the GrA homodimer has a molecular mass above the cutoff (45-50 kDa; Peters, 1984; Jans and Hübner, 1996; see however Ohno et al., 1998) for an NLS requirement for nuclear entry (unlike monomeric $\mathrm{GrB}$ ), this implies that it accumulates through a directed and specific process.

Results were compared to those for the cellular apoptotic cysteine protease CPP32, which did not accumulate in the nucleus or nucleolus (Table 1). Nuclear targeting of GrA was concluded to be specific, and not a general property of proteases involved in apoptosis (see also Trapani et al., 1996). Results were also compared to those for the conventional NLScontaining $\beta$-galactosidase fusion protein $\mathrm{T}$-ag- $\mathrm{CcN}-\beta-\mathrm{Gal}$, which accumulates strongly in the nucleus but not in the nucleolus (see also Jans et al., 1996), and a $70 \mathrm{kDa}$ dextran, which is excluded from the nucleus if the nuclear envelope is intact (Table 1). 
A
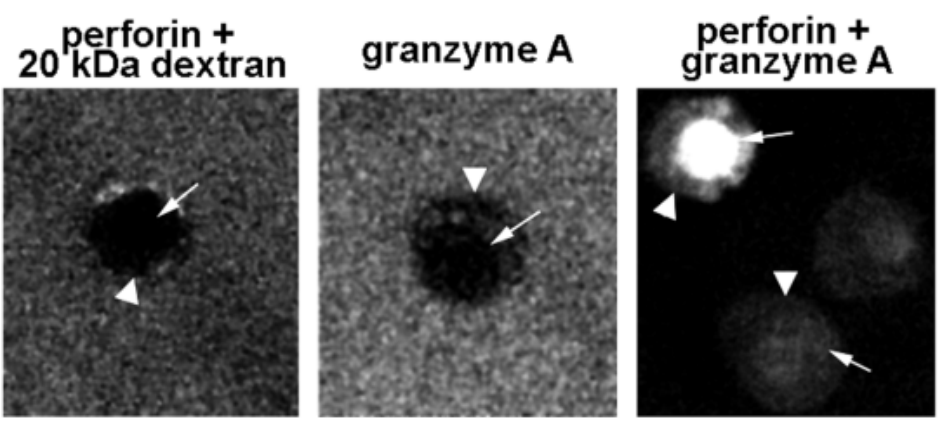

B
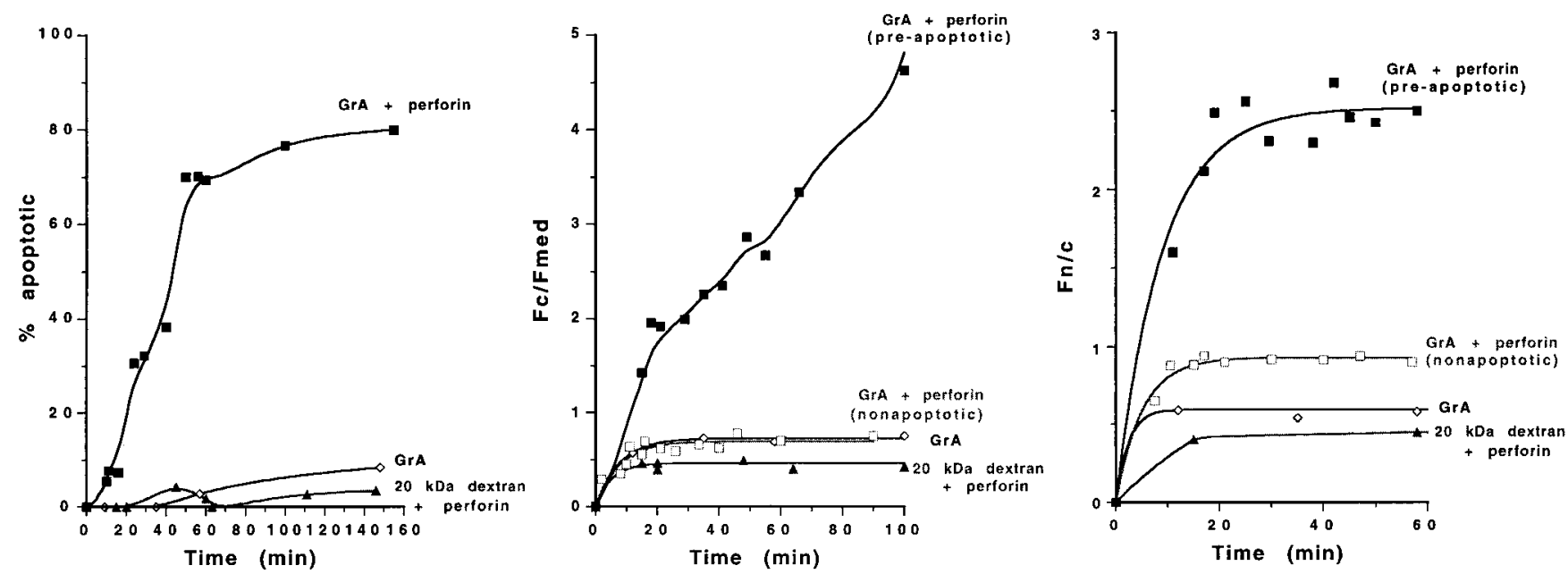

C
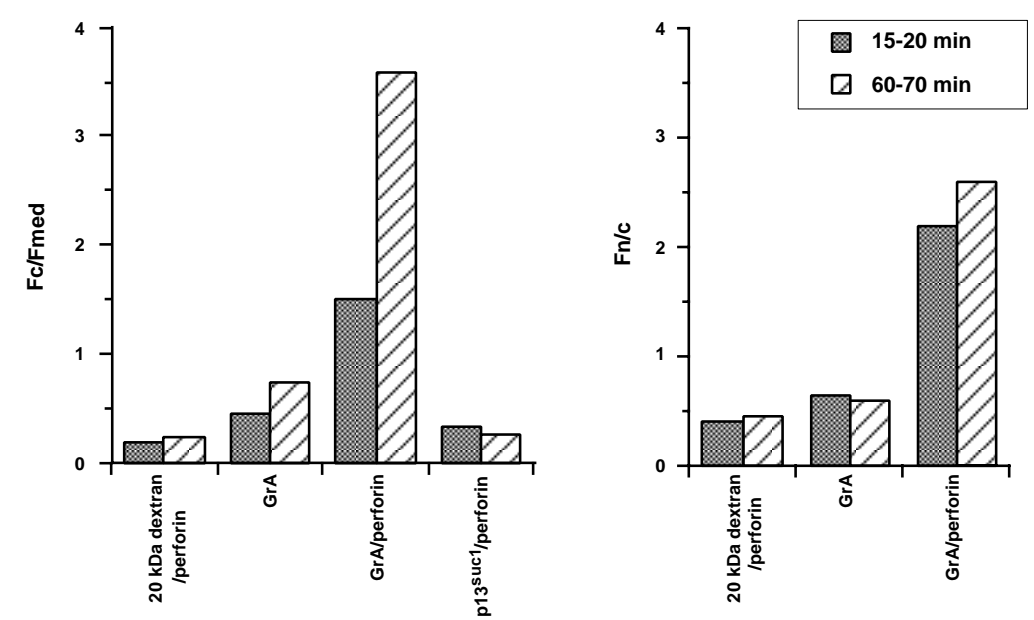

Fig. 1. Visualization and kinetics of the uptake and nuclear accumulation of GrA by FDC-P1 cells in the absence and presence of perforin. (A) Cells were exposed to either FITC-dextran $(20 \mathrm{kDa})$ in the presence of perforin, or FITC-GrA in the absence or presence of perforin at $37^{\circ} \mathrm{C}$ as indicated, and fluorescence visualized after 20-25 minutes using CLSM. The cell membrane perimeter is indicated by an arrowhead, and the nuclear membrane by an arrow. The different cells in the right-most panel are at different stages of apoptosis. (B) Cells were treated as in A, mounted and examined using CLSM. Cells with apoptotic morphology (Trapani et al., 1998) were enumerated visually ( 200 cells per time point) to determine the percentage of pre-apoptotic cells (left panel), and image analysis performed to quantitate either cellular (Fc/Fmedium, middle panel) or nuclear (Fn/c, right panel) uptake (see Materials and methods) for the different cell populations. Results are the average from at least four separate experiments, where the s.e.m. for the measurements from an individual experiment for each value of Fn, Fc and autofluorescence was not greater than $9.2 \%$ the value of the mean. Curve fitting was performed as described previously (Trapani et al., 1998). Maximal nuclear uptake $\left(\mathrm{Fn} / \mathrm{c}_{\max }\right)$ of $\mathrm{GrA}$ in the presence of perforin was 2.52 (regression coefficient $r$ for the curve fit $=0.98$ ) for preapoptotic and $0.93(r=0.99)$ for non-apoptotic cells. Maximal accumulation was achieved in the case of pre-apoptotic cells within 28 minutes ( $\mathrm{t}_{1 / 2}$ was approx. 6 minutes). (C) Cells were treated as in B. The results for GrA in the absence and presence of perforin were compared to those for a $20 \mathrm{kDa}$ FITC-dextran and the $13 \mathrm{kDa}$ cdk subunit $\mathrm{p} 13^{\mathrm{suc} 1}$ in the presence of perforin. Results are averaged from two separate experiments, each individual measurement representing at least five separate measurements for each of Fc, Fmedium, Fn and autofluorescence, where the s.e.m. was not greater than $7.2 \%$ of the mean. 


\section{Lack of dependence of GrA nuclear/nucleolar import on ATP}

NLS-dependent nuclear protein import is an active ATPdependent process (Newmeyer and Forbes, 1988; see Jans and Hübner, 1996). We tested whether this also applied to nuclear/nucleolar accumulation of GrA (Fig. 2) by carrying out apyrase pretreatment of both exogenously added cytosol and perforated cells and omitting the ATP-regenerating system in the assay (see Materials and methods). Surprisingly, this did not affect the maximal extent of nuclear or nucleolar import at all $\left(\mathrm{Fn} / \mathrm{c}_{\max }=4.5\right.$ and $\mathrm{Fnu} / \mathrm{c}_{\max }=5.4$ in the absence of ATP $)$, although nuclear/nucleolar import rates were somewhat reduced (see Table 1). This was in contrast to results for the NLS-containing T-ag-CcN- $\beta$-gal protein (Table 1; Efthymiadis et al., 1997, 1998), whose nuclear import was strongly inhibited by apyrase pretreatment. It was concluded that GrA nuclear/nucleolar accumulation was essentially independent of ATP. Nuclear and nucleolar transport of GrA was also not inhibited by the non-hydrolyzable GTP analog GTP $\gamma$ S (Table 1), in contrast to nuclear import of T-ag-CcN$\beta$-gal (Table 1) and active NLS-dependent nuclear protein import in general (Moore and Blobel, 1994; see Jans and

A

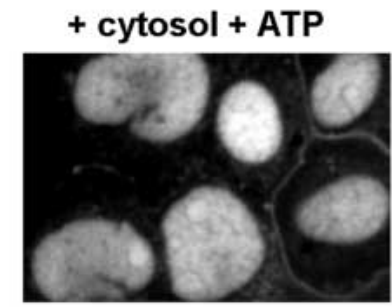

+ cytosol - ATP

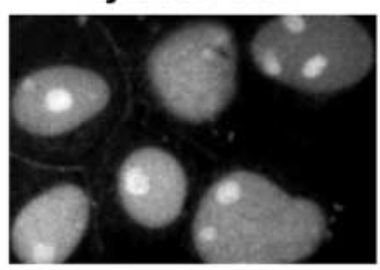

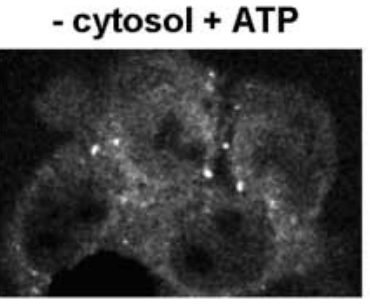

- cytosol - ATP

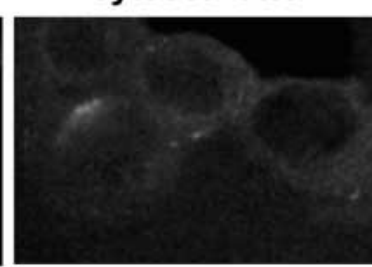

Hübner, 1996). These results indicated that GrA nuclear import was independent of the monomeric GTPase Ran, further supporting the idea that GrA accumulates in the nucleus through a novel nuclear import pathway.

\section{Dependence of GrA nuclear/nucleolar import on cytosolic factors}

Active, NLS-dependent nuclear protein import is known to be dependent on cytosolic factors, including Ran and the NLSbinding importin subunits (Moore and Blobel, 1994; Görlich et al., 1995a,b; see Jans and Hübner, 1996). We tested whether this was the case for GrA nuclear/nucleolar import (Fig. 2) by performing transport assays in the absence of exogenously added cytosol. Nuclear/nucleolar accumulation was severely reduced (Fig. 2A right top panel, B) both in terms of maximal accumulation $\left(70 \%\right.$ reduced for $\mathrm{Fn} / \mathrm{c}_{\max }$ and $50 \%$ reduced for $\left.\mathrm{Fnu} / \mathrm{c}_{\max }\right)$ and import rate (Table 1$)$. A similar reduction in nuclear import in the absence of exogenous cytosol was observed for the conventional NLS-containing T-ag-CcN- $\beta$-gal (Table 1; Jans et al., 1996; Efthymiadis et al., 1997, 1998). GrA nuclear/nucleolar transport was concluded to be dependent on cytosolic factors.
B

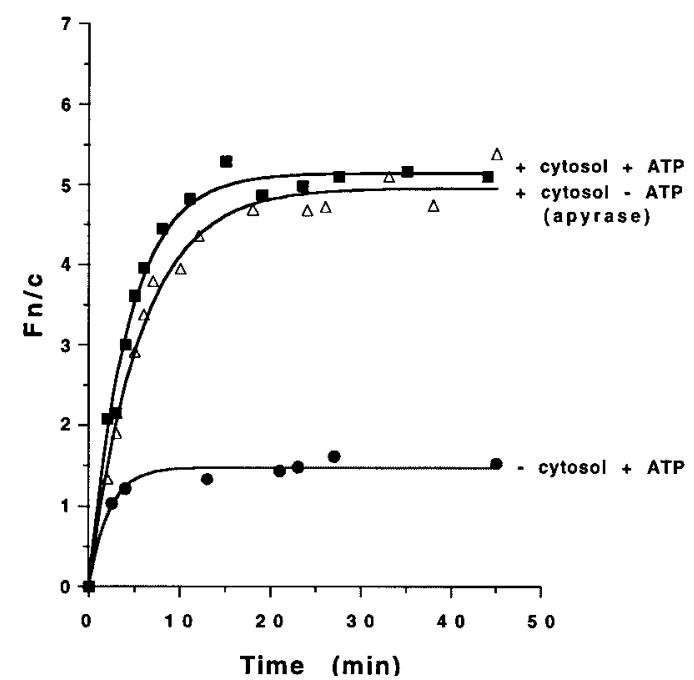

Fig. 2. Visualization and kinetics of nuclear and nucleolar accumulation of GrA in vitro in mechanically perforated HTC cells. (A) Cells were mechanically perforated as described in Materials and methods (Jans et al., 1991), and fluorescence due to nuclear/nucleolar accumulation of GrA in the presence and absence of exogenously added cytosol, and/or ATP as indicated, visualised at 10 minutes using CLSM (oil immersion objective, $\times 60$ ). For experiments in the absence of ATP, cells and cytosol were pretreated with apyrase and measurements performed in the absence of the ATP-regenerating system (see Materials and methods). (B) Cells were treated as in A and nuclear and nucleolar accumulation followed with time using CLSM. Results represent the average for two separate experiments, each point representing the average of at least ten separate measurements for each value of nucleolar fluorescence (Fnu), Fn and Fc respectively, with autofluorescence subtracted. Curves are fitted for the function $F n / c(t)=$ $\mathrm{Fn} / \mathrm{c}_{\max } *\left(1-\mathrm{e}^{-\mathrm{kt}}\right)\left(\right.$ Jans et al., 1991), or Fnu/c(t) $=$ Fnu/c $\max *\left(1-\mathrm{e}^{-\mathrm{kt}}\right)$ (Jans et al., 1996), where $t$ is time in minutes (see Table 1 for pooled data).

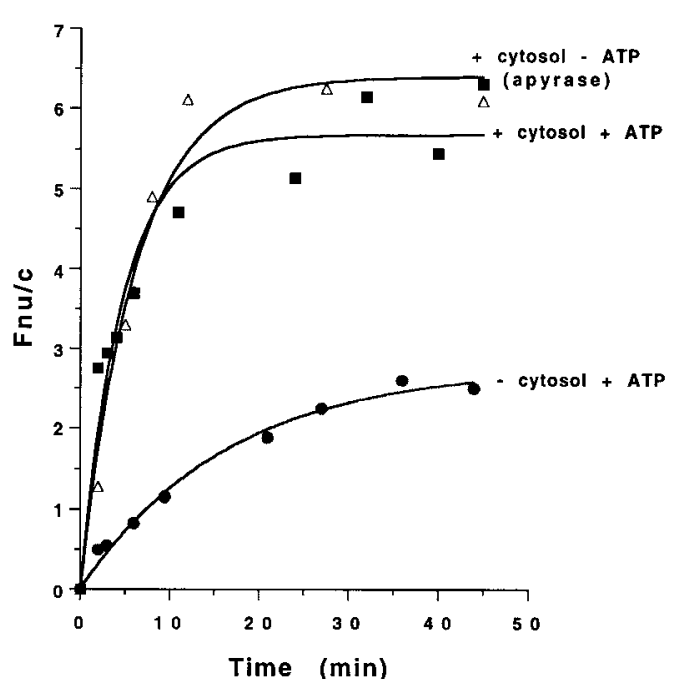


Fig. 3. (A) Nuclear/nucleolar accumulation of granzyme A compared to that of the SV40 T-ag fusion protein T-ag-CcN- $\beta$-Gal in the presence of specific antibodies to nucleolin (C23) or perforin (PB2; negative control) in vitro in mechanically perforated HTC cells. Results are expressed as a percentage of accumulation in the presence of PB2 \pm s.e.m. (B) Nuclear accumulation of a $70 \mathrm{kDa}$ FITC-labelled dextran (indicative of nuclear envelope integrity) and $\mathrm{T}-\mathrm{ag}-\mathrm{CcN}-\beta-\mathrm{Gal}$ (indicative of active NLS-dependent nuclear import) in the absence and presence of exogenously added GrA $(0.5 \mu \mathrm{M})$ in vitro in mechanically perforated HTC cells. Results are for nuclear

exclusion/accumulation in the presence of cytosol and the ATP regenerating system, and represent two separate experiments ( \pm s.e.m.).

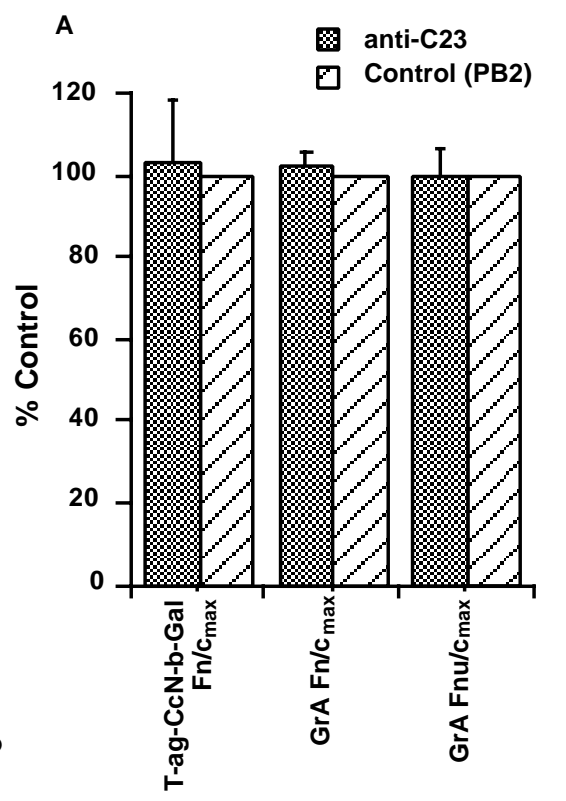

import kinetics of the conventional NLS-containing T-ag-CcN$\beta$-Gal fusion protein, as well as the nuclear exclusion properties of the nuclear envelope with respect to a $70 \mathrm{kDa}$ dextran, in the absence or presence of $0.5 \mu \mathrm{M}$ unlabelled GrA (Fig. 3B). There was no significant difference in either the maximal level of nuclear accumulation of T-ag-CcN- $\beta-\mathrm{Gal}$ in the presence of $\mathrm{GrA}\left(\mathrm{Fn} / \mathrm{c}_{\max }=6.5\right)$, or in its absence $\left(\mathrm{Fn} / \mathrm{c}_{\max }\right.$ $=5.9)$ (Fig. 3B). Similarly, the $70 \mathrm{kDa}$ dextran was excluded from the nuclei in the presence of $\mathrm{GrA}\left(\mathrm{Fn} / \mathrm{c}_{\max }=0.17\right)$ to the same extent as in its absence $\left(\mathrm{Fn} / \mathrm{c}_{\max }=0.14\right)$ (Fig. 3B), indicating intactness of the nuclear envelope. These results indicate that the NPC and nuclear envelope are not direct targets of proteolysis by GrA, and that the cytosolic components of the nuclear import system remain fully functional in the presence of GrA.

\section{Nuclear accumulation of granzymes in the absence of an intact nuclear envelope}

The results for the lack of dependence of GrA nuclear/nucleolar accumulation on ATP and its inability to be inhibited by GTP $\gamma \mathrm{S}$ implied that accumulation may be through binding to nuclear components. To test this, nuclear/nucleolar accumulation was assessed in the presence of the detergent CHAPS, which permeabilises the nuclear envelope; under these conditions, nuclear accumulation can only occur through binding to nuclear components such as chromatin, lamins etc. (see Efthymiadis et al., 1997, 1998). $\mathrm{GrB}$ was also examined in this fashion. Both GrA and GrB were found to accumulate strongly in the nucleus and nucleolus in the presence of CHAPS (Fig. 4; Table 1), irrespective of the addition of cytosol. For comparison, experiments were performed using CPP32, the NLScontaining T-ag-CcN- $\beta$-Gal fusion protein, and a $70 \mathrm{kDa}$ dextran, all of which were found not to accumulate in the nucleus, and to be excluded from the nucleolus, instead equilibrating between cytoplasm and nucleus (Table 1; see Efthymiadis et al., 1997, 1998). Experiments were also performed in the presence of antibodies to $\mathrm{C} 23$ (Fig. 4A bottom panels, B right panel, and not shown), with no evident effect in terms of a decrease in the nuclear/nucleolar accumulation of either granzyme. It was concluded that GrA and $\mathrm{GrB}$ are able to bind to nuclear components and thereby accumulate in the nucleus/nucleolus through a specific process independent of exogenous cytosol in the absence of an intact nuclear envelope. The fact that cytosol is required for nuclear accumulation in the presence of an intact nuclear envelope (Fig. 2; Table 1) indicates that cytosolic factors are specifically required under normal conditions to effect nuclear entry of the granzymes.

\section{DISCUSSION}

This study establishes for the first time that GrA can localize in the nucleus of intact FDC-P1 cells in a perforin-dependent fashion, and is capable of localizing in the nucleus in vitro in mechanically perforated HTC cells in the presence of exogenously added cytosol. That GrA should thus resemble $\mathrm{GrB}$ in this respect is more than surprising in view of their quite divergent structures and substrate specificity, and above-all the fact that GrA is larger than the molecular mass cut-off for entering the nucleus by passive diffusion, whereas $\mathrm{GrB}$ is not. Since GrA and GrB are so different, the fact that they both appear to be targeted to the nucleus in perforin-dependent fashion in intact target cells implies that this represents a common pathway of significance in cytolytic-granulemediated apoptosis. In contrast, the active form of the nongranule apoptotic caspase CPP32 does not accumulate in the nucleus in vitro as shown here, and appears not to translocate to the nucleus even in cells undergoing apoptosis (Liu et al., 1997; see also Krajewski et al., 1997), implying that CPP32 may not be important in direct cleavage of nuclear poly(ADPribose) polymerase (PARP), one of the central targets of apoptotic proteolytic cleavage, in vivo. The serine protease chymotrypsin also does not accumulate in the nucleus (Trapani et al., 1996), consistent with the idea that nuclear targeting may 


\section{A}

Fig. 4. Visualization (A) and quantitation (B) of nuclear and nucleolar accumulation of $\mathrm{GrA}$ and $\mathrm{GrB}$ in vitro in mechanically perforated HTC cells in the presence of the nuclear envelope permeabilizing detergent CHAPS and in the absence and presence of anti-nucleolin (C23) antibody. (A) Cells were mechanically perforated, and accumulation of GrA or GrB in the presence of $0.025 \%$ CHAPS and exogenously added cytosol, in the absence and presence of anti-C23 antibody as indicated, was visualised after 10 minutes using CLSM (oil immersion objective, $\times 60$ ). (B) Cells were treated as in $\mathrm{A}$ and maximal nuclear and nucleolar accumulation determined after 30 minutes. Results represent two separate experiments ( \pm s.e.m.), where each measurement represents the average of at least eight separate measurements for each value of Fnu, Fn and Fc, with autofluorescence subtracted (see Table 1 for pooled data). Results are compared to those for the T-ag-CcN- $\beta-G a l$, a $70 \mathrm{kDa}$ FITC-labelled dextran, and FITC-CPP32 (left panel), and to those for GrA and $\mathrm{GrB}$ in the presence of an anti-C23 antibody (right panel).

\section{+ cytosol + CHAPS \\ + cytosol \\ + CHAPS \\ + anti-C23}
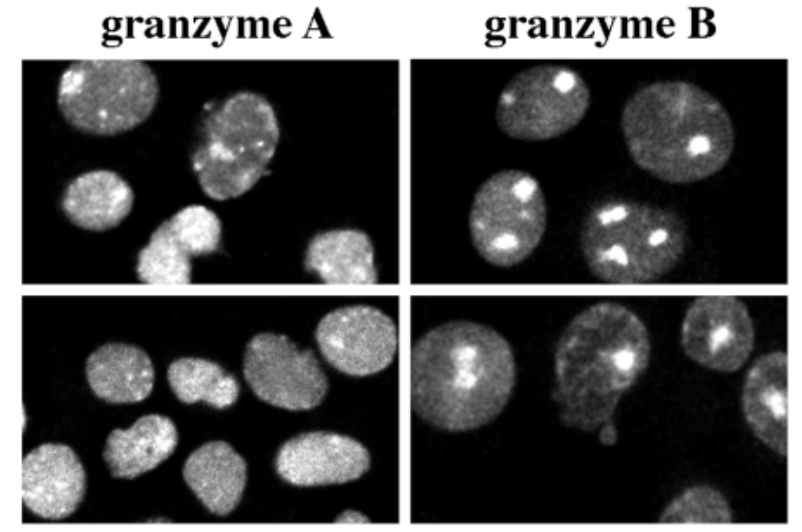

B
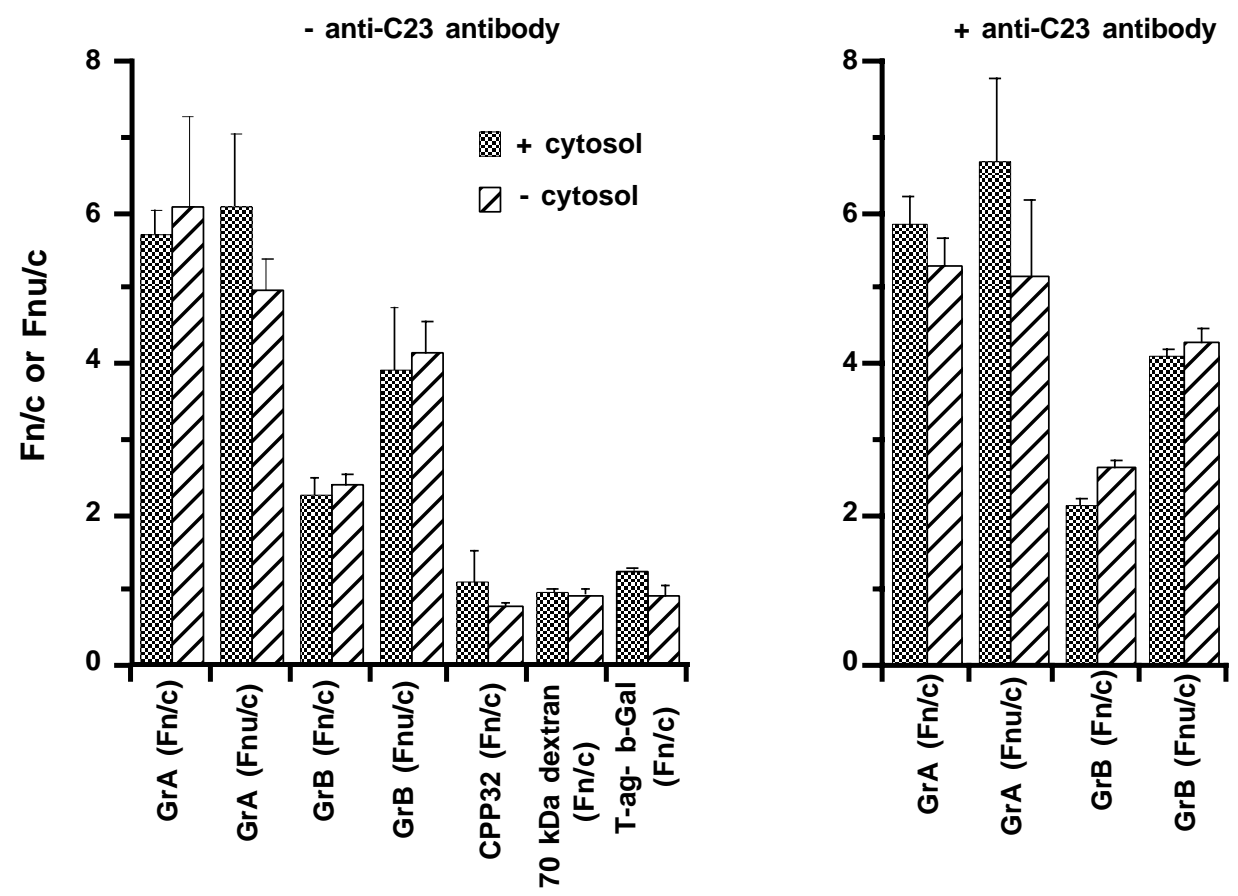

be specific to granzymes, conferring upon them the ability to communicate the apoptotic signal to the nucleus directly.

This study clearly indicates that the cytolytic granule component GrA, like GrB, can enter target cells and accumulate in the nucleus in the presence of perforin, and hence is a protein able to be transported all the way from the external plasma membrane surface to the cell nucleus. How GrA or GrB enter cells remains unclear, but perforin, which does not enter cells (Ortaldo et al., 1992; Shi et al., 1997), is clearly essential for GrA and GrB nuclear accumulation. The specificity of granzyme uptake and nuclear accumulation in the presence of perforin is demonstrated by the fact that neither a $20 \mathrm{kDa}$ dextran nor the $13 \mathrm{kDa}$ protein $\mathrm{p} 13^{\text {suc } 1}$ are able to enter intact cells in the presence of perforin. The nature of the membrane signalling event effected by perforin is not clear, but one possibility is that granzymes taken up by intact cells may be in a vesicular or complexed form which cannot access the cytoplasm, and requires perforin-induced signalling to enable it to access the cytoplasm prior to targeting to the nucleus
(Froehlich et al., 1996b). An alternative is that perforin triggers cytosolic phosphorylation or some other activity necessary to effect granzyme nuclear uptake in intact cells, the exogenously added cytosol necessary for $\mathrm{GrA} / \mathrm{GrB}$ nuclear accumulation in vitro possibly being able to substitute this in the perforated cell system.

Although nuclear and nucleolar transport of GrA in vitro was found to be dependent on cytosolic factors, it was independent of ATP and not inhibited by a non-hydrolyzable GTP analog, and thus clearly independent of both Ran (Moore and Blobel, 1994) and as yet unidentified GTPases with a proposed role in nuclear import (see Takei et al., 1994; Sweet and Gerace, 1996). Thus, despite being above the 45-50 kDa size cut-off for free passage into and out of the nucleus via diffusion (Peters, 1984; Jans and Hübner, 1996; see however Ohno et al., 1998), GrA clearly does not accumulate in the nucleus through a 'classical' NLS-dependent active pathway, and consistent with this it is not recognized by the NLS-binding importin subunits. Indeed, the ability of GrA and GrB to accumulate in the nucleus in the 
presence of the nuclear envelope-permeabilizing detergent CHAPS, as shown here for the first time, indicates that both accumulate in the nucleus through binding to nuclear components, independent of the presence of cytosolic factors. Our experiments designed to assess granzyme nuclear accumulation in the presence of specific antibodies to nucleolin indicate that the latter is unlikely to play a role in granzyme nuclear/nucleolar accumulation, although a protein with similar properties to nucleolin (see Xue et al., 1993) could well play a role as a transporter ferrying GrA/GrB from the cytoplasm to the nucleus/nucleolus. A candidate for the latter might be the $69 \mathrm{kDa}$ cytosolic protein identified by Pinkoski et al. (1996) as binding GrB in situ, whilst a $80 \mathrm{kDa}$ nuclear protein recognised by $\mathrm{GrB}$ that is strongly associated with heterochromatin (see Pinkoski et al., 1996) may well constitute the binding site for granzymes in the nucleus; whether these molecules are also recognized by GrA remains to be demonstrated.

The cellular consequences of exposure to perforin and GrA or $\mathrm{GrB}$ are nuclear changes that reflect apoptosis. Whilst perforin acts exclusively at the target cell membrane (Ortaldo et al., 1992; Trapani et al., 1996) to provide an apparently additional but essential signal in intact cells, this and recent studies (Trapani et al., 1996; Pinkoski et al., 1996; Jans et al., 1996) indicate that GrA and GrB can target to the nucleus both in vitro and in intact cells in the presence of perforin, implying that granzymes can directly contribute to the initiation of DNA fragmentation. Although the distinctive nuclear changes during apoptosis include disassembly/dissolution of the nuclear lamina and envelope, we show here that the active transport and passive permeability properties of the nucleus are completely retained in the presence of unlabelled GrA, indicating intactness both of the nuclear envelope and the cellular nuclear transport system. Accordingly, we may conclude that the components mediating nuclear protein import, as well as the nuclear envelope and NPC, are not targets of GrA proteolytic activity, the nature of which can only be surmised at this stage, although GrB has been shown to be able to cleave resident nuclear proteins such as PARP (Froehlich et al., 1996a) and the double stranded DNA-dependent protein kinase catalytic subunit (Song et al., 1996) in vitro.

In view of the results reported here and elsewhere (Jans et al., 1996; Pinkoski et al., 1996; Trapani et al., 1996, 1998; Shi et al., 1997), it seems clear that perforin-dependent nuclear targeting of granzymes has an important role in cytolytic granule-mediated apoptosis. Questions that remain include identifying the key substrates of nuclear localized $\mathrm{GrA} / \mathrm{GrB}$, as well as determining the precise nature of the membrane signal elicited by perforin, which induces granzyme translocation from the cytoplasm to the nucleus as part of a unique nuclear import pathway. Future work in this laboratory is focussed on both of these aspects.

This work was supported by a Clive and Vera Ramaciotti Foundation Grant to D. A. J., and a project grant and senior research fellowship from the National Health and Medical Research Council of Australia to J. A. T.

\section{REFERENCES}

Adam, S.A., Sterne-Marr, R. E. and Gerace, L. (1990). Nuclear import in permeabilized mammalian cells requires soluble cytoplasmic factors. J. Cell Biol. 111, 807-816.
Adam, S.A. and Gerace, L. (1991). Cytosolic factors that specifically bind nuclear location signals are receptors for nuclear transport. Cell $\mathbf{6 6}, 837$ 847.

Borer, R. A., Lehner, C. F., Eppenberger, H. M. and Nigg, E. A. (1989). Major nucleolar proteins shuttle between nucleus and cytoplasm. Cell 56, 379-390.

Earnshaw, W. C. (1995). Nuclear changes in apoptosis. Curr. Opin. Cell Biol. 7, 337-343.

Ebnet, K., Hausmann, M., Lehmann-Grube, F., Muellbacher, A., Kopf, M., Lamers, M. and Simon, M. M. (1995). Granzyme A-deficient mice retain potent cell-mediated cytotoxicity. EMBO J. 14, 4230-4239.

Efthymiadis, A., Briggs, L. J. and Jans, D. A. (1998). The HIV-1 Tat nuclear localization sequence confers novel nuclear import properties. J. Biol. Chem. 273, 1623-1628.

Efthymiadis, A., Shao, H., Hübner, S. and Jans, D. A. (1997). Kinetic characterization of the human retinoblastoma protein bipartite nuclear localization signal in vivo and in vitro: a comparison with the SV40 large T-antigen NLS. J. Biol. Chem. 272, 22134-22139.

Froelich, C. J., Hanna, W. L., Poirier, G. G., Duriez, P. J., D'Amours, D., Salvesan, G. S., Alnemri, E. S., Earnshaw, W. C. and Shah, G. M. (1996a). Granzyme B/perforin mediated apoptosis of Jurkat cell results in cleavage of ploy(ADPribose) polymerase to the $89 \mathrm{kDa}$ apoptotic fragment and a less abundant $64 \mathrm{kDa}$ fragment. Biochem. Biophy. Res. Commun. 227, 658-667.

Froelich, C. J., Orth, K., Turbov, J., Seth, P., Gottlieb, R., Babior, B., Shah, G. M., Bleackley, R. C., Dixit, V. M. and Hanna, W. (1996b). New paradigm for lymphocyte granule mediated cytotoxicity: target cells bind and internalize granzyme B but an endosomolytic agent is necessary for cytosolic delivery and subsequent apoptosis. J. Biol. Chem. 271, 2907329079.

Froelich, C. J., Turbov, J. and Hanna, W. (1996c). Human perforin: rapid enrichment by immobilized metal affinity chromatography. Biochem. Biophy. Res. Commun. 229, 44-49.

Görlich, D., Kostka, S., Kraft, R., Dingwall, C., Laskey, R. A., Hartmann, E. and Prehn, S. (1995a). Two different subunits of importin cooperate to recognize nuclear localization signals and bind them to the nuclear envelope. Curr. Biol. 5, 383-392.

Görlich, D., Vogel, F., Mills, A. D., Hartmann, E. and Laskey, R. A. (1995b). Distinct functions for the two importin subunits in nuclear protein import. Nature 377, 246-248.

Hanna, W., Zhang, X., Turbov, J., Winkler, U., Hudig, D. and Froelich, C. J. (1996). Purification of Cationic Granule Proteases: Application to Human Granzymes. In Protein Expression and Purification 4, 398-404.

Harvey, N. L., Butt, A. J. and Kumar, S. (1997). Functional activation of Nedd2/ICH-1 (caspase-2) is an early process in apoptosis. J. Biol. Chem. 272, 13134-13139.

Hayes, M. P., Berrebi, G. A. and Henkart, P. A. (1989). Induction of target cell DNA release by cytotoxic T lymphocyte granule protease granzyme A J. Exp. Med. 170, 933-946.

Henkart, P. A., Millard, P. J., Reynolds, C. W. and Henkart, P. A. (1984). Cytolytic activity of purified cytoplasmic granules from cytotoxic rat large granular lymphocyte tumors. J. Exp. Med. 160, 75-93.

Heusel, J. W., Wesselschmidt, R. L., Shresta, S., Russell, J. H. and Ley, T. J. (1994). Cytotoxic lymphocytes require granzyme B for the rapid induction of DNA fragmentation and apoptosis in allogeneic target cells. Cell 76, 977-987.

Hübner, S., Xiao, C.-Y. and Jans, D. A. (1997). The protein kinase CK2 site (Ser ${ }^{11 / 112}$ ) enhances recognition of the SV40 large T-antigen nuclear localization sequence by importin subunits. J. Biol. Chem. 272, 1719117195.

Irmler, M., Hertig, S., MacDonald, H. R., Sadoul, R., Becherer, D., Proudfoot, A., Solari, R. and Tschopp, J. (1995). Granzyme A is an interleukin 1ß-converting enzyme. J. Exp. Med. 181, 1917-1922.

Jans, D. A., Ackermann, M., Bischoff, J. R., Beach, D. H. and Peters, R. (1991). p34 $4^{\text {cdc2 }}$-mediated phosphorylation at $\mathrm{T}^{124}$ inhibits nuclear import of SV40 T-antigen proteins. J. Cell Biol. 115, 1203-1212.

Jans, D. A. and Hübner, S. (1996). Regulation of protein transport to the nucleus - the central role of phosphorylation. Physiol. Rev. 76, 651-685.

Jans, D. A., Jans, P., Briggs, L. J., Sutton, V. and Trapani, J. A. (1996). Nuclear transport of granzyme B (fragmentin-2) in vitro - dependence on cytosolic factors. J. Biol. Chem. 271, 30781-30789.

Karasuyama, H. and Melchers, F. (1988). Establishment of mouse cell lines which constitutively secrete large quantities of interleukin $2,3,4$ or 5, using modified cDNA expression vectors. Eur. J. Immunol. 18, 97-102. 
Krajewski, S., Gasgoyne, R. D., Zapata, J. M., Krajewski, M., Kitada, S., Chhanabhal, M., Horsman, D., Berean, K., Piro, L. D., Fugier-Vivier, I., Liu, Y. J., Wang, H.-C. and Reed, J. C. (1997). Immunolocalization of the ICE/Ced-3-family protease, CPP32 (Caspase-3), in non-Hodgkin's lymphomas, chronic lymphocytic leukemias, and reactive lymph nodes. Blood 89, 3817-3825.

Liu, X., Zou, H., Slaughter, C. and Wang, X. (1997). DFF, a heterodimeric protein that functions downstream of Caspase-3 to trigger DNA fragmentation during apoptosis. Cell 89, 175-184.

Moore, M. S. and Blobel, G. (1994). A G protein involved in nucleocytoplasmic transport: the role of Ran. Trends Biochem. Sci. 19, 211216.

Munger, W. E., Berrebi, G. A. and Henkart, P. A. (1988). Possible involvement of CTL granule proteases in target cell DNA breakdown. Immunol. Rev. 103, 99-109.

Newmeyer, D. D. and Forbes, D. J. (1988). Nuclear import can be separated into distinct steps in vitro: Nuclear pore binding and translocation. Cell 52, 641-653.

Ohno, M., Fornerod, M. and Mattaj, I. W. (1998). Nucleocytoplasmic transport: the last 200 nanometers. Cell 92, 327-336.

Ortaldo, J. R., Winkler-Pickett, R. T., Nagashima, K., Yagita, H. and Okumura, K. (1992). Direct evidence for release of pore-forming protein during NK cellular lysis. J. Leuk. Biol. 52, 483-488.

Pasternack, M. S., Bleier, K. J. and McInerney, T. N. (1991). Granzyme A binding to target cell proteins. Granzyme A binds to and cleaves nucleolin in vitro. J. Biol. Chem. 266, 14703-14708.

Peters, R. (1984). Nucleo-cytoplasmic flux and intracellular mobility in single hepatocytes measured by fluorescence microphotolysis. EMBO J. 3, 18311836.

Pinkoski, M. J., Winkler, U., Hudig, D. and Bleackley, R. C. (1996). Binding of granzyme B in the nucleus of target cells. Recognition of the 80 kilodalton protein. J. Biol. Chem. 271, 10225-10229.

Poe, M., Blake, J. T., Boulton, D. A., Gammon, M., Sigal, N. H., Wu, J. K. and Zweerink, H. J. (1991). Human cytotoxic lymphocyte granzyme B. Its purification from granules and the characterization of substrate and inhibitor specificity. J. Biol. Chem. 266, 98-103.

Rihs, H.-P., Jans, D. A., Fan, H. and Peters, R. (1991). The rate of nuclear cytoplasmic protein transport is determined by the casein kinase II site flanking the nuclear localization sequence of SV40 T-antigen. EMBO J. 10, 633-639.
Shi, L., Kraut, R. P., Aebersold, R. and Greenberg, A. H. (1992a). A natural killer cell granule protein that induces DNA fragmentation and apoptosis. J. Exp. Med. 175, 553-565.

Shi, L., Kam, C.-M., Powers, J. C., Aebersold, R. and Greenberg, A. H. (1992b). Purification of three cytotoxic lymphocyte granule serine proteases that induce apoptosis through distinct substrate and target cell interactions.J. Exp. Med. 176, 1521-1529.

Shi, L., Mai, S., Israels, S., Browne, K., Trapani, J. A. and Greenberg, A. H. (1997). Granzyme B autonomously crosses the cell membrane and perforin initiates apoptosis and granyzme B nuclear accumulation. J. Exp. Med. 186, 855-866.

Song, Q., Burrows, S., Lees-Miller, S., Smith, G., Jackson, S., Kumar, S., Trapani, J. A., Alnemri, E., Litwack, G., Lu, H., Moss, D. and Lavin, M. (1996). ICE-like protease cleaves DNA-dependent protein kinase in cytotoxic T-cell killing.J. Exp. Med. 184, 619-628.

Suidan, H. S., Bouvier, J., Schaerer, E., Stone, S. R., Monard, D. and Tschopp, J. (1994). Granzyme A released upon stimulation of $T$ lymphocytes activates the thrombin receptor on neuronal cells and astrocytes. Proc. Nat. Acad. Sci. USA 91, 8112-8116.

Sweet, D. J., and Gerace, L. (1996). A GTPase distinct from Ran is involved in nuclear protein import. J. Cell Biol. 133, 971-983.

Takei, Y., Takahashi, K. Kanaho, Y. and Kanada, T. (1994). Possible involvement of a pertussin toxin-sensitive GTP-binding protein in protein transport into nuclei isolated from rat liver. J. Biochem. 115, 578583.

Trapani, J. A., Browne, K. A., Dawson, M. and Smyth, M. J. (1993). Purification of functional asp-ase (natural killer granzyme B) using a monoclonal antibody. Biochem. Biophys. Research Commun. 158, 910920.

Trapani, J. A., Browne, K. A., Smyth, M. J. and Jans, D. A. (1996). Localization of granzyme B in the nucleus: a putative role in the mechanism of cytotoxic lymphocyte-mediated apoptosis. J. Biol. Chem. 271, 41274133.

Trapani, J. A., Jans, P., Smyth, M. J. Froelich, C. J., Williams, E. A., Sutton, V. R. and Jans, D. A. (1998). Perforin-dependent nuclear entry of granzyme B precedes apoptosis, and is not a consequence of nuclear membrane dysfunction. Cell Death Differ. 5, 488-496.

Xue, Z., Shan, X., Lapayre, B. and Melese, T. (1993). The amino terminus of mammalian nucleolin specifically recognises SV40 T-antigen-type nuclear localization sequences. Eur. J. Cell Biol. 62, 13-21. 